\title{
ON 2-ABSORBING IDEALS OF COMMUTATIVE RINGS
}

\author{
AYMAN BADAWI
}

\begin{abstract}
Suppose that $R$ is a commutative ring with $1 \neq 0$. In this paper, we introduce the concept of 2-absorbing ideal which is a generalisation of prime ideal. A nonzero proper ideal $I$ of $R$ is called a 2-absorbing ideal of $R$ if whenever $a, b, c \in R$ and $a b c \in I$, then $a b \in I$ or $a c \in I$ or $b c \in I$. It is shown that a nonzero proper ideal $I$ of $R$ is a 2-absorbing ideal if and only if whenever $I_{1} I_{2} I_{3} \subseteq I$ for some ideals $I_{1}, I_{2}, I_{3}$ of $R$, then $I_{1} I_{2} \subseteq I$ or $I_{2} I_{3} \subseteq I$ or $I_{1} I_{3} \subseteq I$. It is shown that if $I$ is a 2-absorbing ideal of $R$, then either $\operatorname{Rad}(I)$ is a prime ideal of $R$ or $\operatorname{Rad}(I)=P_{1} \cap P_{2}$ where $P_{1}, P_{2}$ are the only distinct prime ideals of $R$ that are minimal over $I$. Rings with the property that every nonzero proper ideal is a 2-absorbing ideal are characterised. All 2-absorbing ideals of valuation domains and Prüfer domains are completely described. It is shown that a Noetherian domain $R$ is a Dedekind domain if and only if a 2-absorbing ideal of $R$ is either a maximal ideal of $R$ or $M^{2}$ for some maximal ideal $M$ of $R$ or $M_{1} M_{2}$ where $M_{1}, M_{2}$ are some maximal ideals of $R$. If $R_{M}$ is Noetherian for each maximal ideal $M$ of $R$, then it is shown that an integral domain $R$ is an almost Dedekind domain if and only if a 2-absorbing ideal of $R$ is either a maximal ideal of $R$ or $M^{2}$ for some maximal ideal $M$ of $R$ or $M_{1} M_{2}$ where $M_{1}, M_{2}$ are some maximal ideals of $R$.
\end{abstract}

\section{INTRODUCTION}

We assume throughout that all rings are commutative with $1 \neq 0$. Suppose that $R$ is a ring. Then $T(R)$ denotes the total quotient ring of $R, \operatorname{Nil}(R)$ denotes the set of nilpotent elements of $R, Z(R)$ denotes the set of zerodivisors of $R$, and if $I$ is a proper ideal of $R$, then $\operatorname{Rad}(I)$ denotes the radical ideal of $I$. We start by recalling some background material. A nonzero proper ideal $I$ of a ring $R$ is said to be $Q$-primal if $Z(R / I)=Q / I$ for some prime ideal $Q$ of $R$ containing $I$. A prime ideal $P$ of a ring $R$ is said to be a divided prime ideal if $P \subset(x)$ for every $x \in R \backslash P$; thus a divided prime ideal is comparable to every ideal of $R$. An integral domain $R$ is said to be a divided domain if every prime ideal of $R$ is a divided prime ideal. An integral domain $R$ is said to be a valuation domain if $x \mid y$ (in $\mathrm{R}$ ) or $y \mid x$ (in $\mathrm{R}$ ) for every nonzero $x, y \in R$. It is

Received 31st October, 2007

I would like to thank Professor David F. Anderson and the editors for their great effort in proofreading the manuscript.

Copyright Clearance Centre, Inc. Serial-fee code: 0004-9727/07 \$A2.00+0.00. 
known that a valuation domain is a divided domain. If $I$ is a nonzero ideal of a ring $R$, then $I^{-1}=\{x \in T(R) \mid x I \subseteq R\}$. An integral domain $R$ is called a Prüfer domain if $I I^{-1}=R$ for every nonzero finitely generated ideal $I$ of $R$. An integral domain $R$ is said to be a Dedekind domain if $I I^{-1}=R$ for every nonzero ideal $I$ of $R$. An integral domain $R$ is called an almost Dedekind domain if $R_{M}$ is a Dedekind domain for each maximal ideal $M$ of $R$.

In this paper, we introduce the concept of 2-absorbing ideal which is a generalisation of prime ideal. A nonzero proper ideal $I$ of $R$ is called a 2-absorbing ideal of $R$ if whenever $a, b, c \in R$ and $a b c \in I$, then $a b \in I$ or $a c \in I$ or $b c \in I$. A more general concept than 2 -absorbing ideals is the concept of k-absorbing ideals. We only state the definition of k-absorbing ideals. Suppose that $k$ is a positive integer such that $k>2$. A nonzero proper ideal $I$ of $R$ is called a $k$-absorbing ideal of $R$ if whenever $a_{1}, a_{2}, \ldots, a_{k} \in R$ and $a_{1} a_{2} \cdots a_{k} \in I$, then there are $(\mathrm{k}-1)$ of the $a_{i}$ 's whose product is in $I$. It is easily proved that a nonzero proper ideal $I$ of a principal ideal domain $R$ is a 2-absorbing ideal of $R$ if and only if $I$ is a prime ideal or $I=p^{2} R$ for some prime element $p$ of $R$ or $I=p_{1} p_{2} R$ where $p_{1}, p_{2}$ are distinct prime elements of $R$. Also, it is easily proved that if $P$ and $Q$ are some nonzero prime ideals of a ring $R$, then $P \cap Q$ is a 2-absorbing ideal of $R$. For nontrivial 2-absorbing ideals see Example 2.11, Example 2.12, Example 3.5, and Example 3.11 .

Among many results in this paper, it is shown (Theorem 2.13) that a nonzero proper ideal $I$ of $R$ is a 2-absorbing ideal if and only if whenever $I_{1} I_{2} I_{3} \subseteq I$ for some ideals $I_{1}, I_{2}, I_{3}$ of $R$, then $I_{1} I_{2} \subseteq I$ or $I_{2} I_{3} \subseteq I$ or $I_{1} I_{3} \subseteq I$. It is shown (Theorem 2.4) that if $I$ is a 2-absorbing ideal of $R$, then either $\operatorname{Rad}(I)$ is a prime ideal of $R$ or $\operatorname{Rad}(I)=P_{1} \cap P_{2}$ where $P_{1}, P_{2}$ are the only distinct prime ideals of $R$ that are minimal over $I$. Rings with the property that every nonzero proper ideal is a 2-absorbing ideal are characterised in Theorem 3.4. It is shown (Corollary 2.7) that a 2-absorbing ideal of a ring $R$ is a $Q$ primal ideal for some prime ideal $Q$ of $R$. An example of a $Q$-primal ideal that is not a 2-absorbing ideal is illustrated in Example 3.12. For a valuation domain $R$, it is shown (Proposition 3.10) that a nonzero proper ideal $I$ of $R$ is a 2-absorbing ideal if and only $I=P$ or $I=P^{2}$ where $P=\operatorname{Rad}(I)$ is a prime ideal of $R$. For a Prüfer domain $R$, it is shown (Theorem 3.14) that a nonzero proper ideal $I$ of $R$ is a 2-absorbing ideal if and only if $I$ is a prime ideal of $R$ or $I=P^{2}$ is a $P$-primary ideal of $R$ or $I=P_{1} \cap P_{2}$ where $P_{1}$ and $P_{2}$ are nonzero prime ideals of $R$. It is shown (Corollary 3.16) that a Noetherian domain $R$ that is not a field is a Dedekind domain if and only if a 2-absorbing ideal of $R$ is either a maximal ideal of $R$ or $M^{2}$ for some maximal ideal $M$ of $R$ or $M_{1} M_{2}$ where $M_{1}, M_{2}$ are some maximal ideals of $R$. If $R_{M}$ is Noetherian for each maximal ideal $M$ of an integral domain $R$, then it is shown (Proposition 3.17) that $R$ is an almost Dedekind domain if and only if a 2-absorbing ideal of $R$ is either a maximal ideal of $R$ or $M^{2}$ for some maximal ideal $M$ of $R$ or $M_{1} M_{2}$ where $M_{1}, M_{2}$ are some maximal ideals of $R$. It is 
shown (Theorem 3.6) that if $P$ is a divided prime ideal of a ring $R$ and $I$ is an ideal of $R$ such that $\operatorname{Rad}(I)=P$, then $I$ is a 2-absorbing ideal of $R$ if and only if $I$ is a P-primary ideal of $R$ such that $P^{2} \subseteq I$.

\section{BASIC PROPERTIES OF 2-ABSORBING IDEALS}

TheOREM 2.1. Suppose that $I$ is a 2-absorbing ideal of a ring $R$. Then $\operatorname{Rad}(I)$ is a 2-absorbing ideal of $R$ and $x^{2} \in I$ for every $x \in \operatorname{Rad}(I)$.

Proof: Since $I$ is a 2-absorbing ideal of $R$, observe that $x^{2} \in I$ for every $x \in \operatorname{Rad}(I)$. Let $x, y, z \in R$ such that $x y z \in \operatorname{Rad}(I)$. Then $(x y z)^{2}=x^{2} y^{2} z^{2} \in I$. Since $I$ is a 2absorbing ideal, we may assume that $x^{2} y^{2} \in I$. Since $(x y)^{2}=x^{2} y^{2} \in I, x y \in \operatorname{Rad}(I)$.

We recall the following lemma.

LemMa 2.2. ([4, Theorem 2.1, p. 2]).

Let $I \subseteq P$ be ideals of a ring $R$, where $P$ is a prime ideal. Then the following statements are equivalent:

(1) $P$ is a minimal prime ideal of $I$;

(2) For each $x \in P$, there is a $y \in R \backslash P$ and a nonnegative integer $n$ such that $y x^{n} \in I$.

Theorem 2.3. Suppose that $I$ is a 2-absorbing ideal of a ring $R$. Then there are at most two prime ideals of $R$ that are minimal over $I$.

Proof: Suppose that $J=\left\{P_{i} \mid P_{i}\right.$ is a prime ideal of $R$ that is minimal over $\left.I\right\}$ and suppose that $J$ has at least three elements. Let $P_{1}, P_{2} \in J$ be two distinct prime ideals. Hence there is an $x_{1} \in P_{1} \backslash P_{2}$, and there is an $x_{2} \in P_{2} \backslash P_{1}$. First we show that $x_{1} x_{2} \in I$. By Lemma 2.2, there is a $c_{2} \notin P_{1}$ and a $c_{1} \notin P_{2}$ such that $c_{2} x_{1}^{n} \in I$ and $c_{1} x_{2}^{m} \in I$ for some $n, m \geqslant 1$. Since $x_{1}, x_{2} \notin P_{1} \cap P_{2}$ and $I$ is a 2-absorbing ideal of $R$, we conclude that $c_{2} x_{1} \in I$ and $c_{1} x_{2} \in I$. Since $x_{1}, x_{2} \notin P_{1} \cap P_{2}$ and $c_{2} x_{1}, c_{1} x_{2} \in I \subseteq P_{1} \cap P_{2}$, we conclude that $c_{2} \in P_{2} \backslash P_{1}$ and $c_{1} \in P_{1} \backslash P_{2}$, and thus $c_{1}, c_{2} \notin P_{1} \cap P_{2}$. Since $c_{2} x_{1} \in I$ and $c_{1} x_{2} \in I$, we have $\left(c_{1}+c_{2}\right) x_{1} x_{2} \in I$. Observe that $c_{1}+c_{2} \notin P_{1}$ and $c_{1}+c_{2} \notin P_{2}$. Since $\left(c_{1}+c_{2}\right) x_{1} \notin P_{2}$ and $\left(c_{1}+c_{2}\right) x_{2} \notin P_{1}$, we conclude that neither $\left(c_{1}+c_{2}\right) x_{1} \in I$ nor $\left(c_{1}+c_{2}\right) x_{2} \in I$, and hence $x_{1} x_{2} \in I$. Now suppose there is a $P_{3} \in J$ such that $P_{3}$ is neither $P_{1}$ nor $P_{2}$. Then we can choose $y_{1} \in P_{1} \backslash\left(P_{2} \cup P_{3}\right), y_{2} \in P_{2} \backslash\left(P_{1} \cup P_{3}\right)$, and $y_{3} \in P_{3} \backslash\left(P_{1} \cup P_{2}\right)$. By the previous argument $y_{1} y_{2} \in I$. Since $I \subseteq P_{1} \cap P_{2} \cap P_{3}$ and $y_{1} y_{2} \in I$, we conclude that either $y_{1} \in P_{3}$ or $y_{2} \in P_{3}$ which is a contradiction. Hence $J$ has at most two elements and that completes the proof.

THEOREM 2.4. Let $I$ be a 2-absorbing ideal of $R$. Then one of the following statements must hold:

(1) $\operatorname{Rad}(I)=P$ is a prime ideal of $R$ such that $P^{2} \subseteq I$. 
(2) $\operatorname{Rad}(I)=P_{1} \cap P_{2}, P_{1} P_{2} \subseteq I$, and $\operatorname{Rad}(I)^{2} \subseteq I$ where $P_{1}, P_{2}$ are the only distinct prime ideals of $R$ that are minimal over $I$.

Proof: By Theorem 2.3, we conclude that either $\operatorname{Rad}(I)=P$ is a prime ideal of $R$ or $\operatorname{Rad}(I)=P_{1} \cap P_{2}$, where $P_{1} P_{2}$ are the only distinct prime ideals of $R$ that are minimal over $I$. Suppose that $\operatorname{Rad}(I)=P$ is a prime ideal of $R$. Let $x, y \in P$. By Theorem 2.1, we have $x^{2}, y^{2} \in I$. Now $x(x+y) y \in I$. Since $I$ is a 2-absorbing ideal, we have $x(x+y)=x^{2}+x y \in I$ or $(x+y) y=x y+y^{2} \in I$ or $x y \in I$. It is easily proved that each case implies that $x y \in I$, and thus $P^{2} \subseteq I$.

Now suppose that $\operatorname{Rad}(I)=P_{1} \cap P_{2}$, where $P_{1} P_{2}$ are the only distinct prime ideals of $R$ that are minimal over $I$. Let $x, y \in \operatorname{Rad}(I)$. Then $x y \in I$ by the same argument given above, and hence $\operatorname{Rad}(I)^{2} \subseteq I$. Now we show that $P_{1} P_{2} \subseteq I$. First observe that $w^{2} \in I$ for each $w \in \operatorname{Rad}(I)$ by Theorem 2.1. Let $x_{1} \in P_{1} \backslash P_{2}$ and $x_{2} \in P_{2} \backslash P_{1}$. Then $x_{1} x_{2} \in I$ by the proof of Theorem 2.3. Let $z_{1} \in \operatorname{Rad}(I)$ and $z_{2} \in P_{2} \backslash P_{1}$. Pick $y_{1} \in P_{1} \backslash P_{2}$. Then $y_{1} z_{2} \in I$ by the proof of Theorem 2.3 and $z_{1}+y_{1} \in P_{1} \backslash P_{2}$. Thus $z_{1} z_{2}+y_{1} z_{2}=\left(z_{1}+y_{1}\right) z_{2} \in I$, and hence $z_{1} z_{2} \in I$. A similar argument will show that if $z_{1} \in \operatorname{Rad}(I)$ and $z_{2} \in P_{1} \backslash P_{2}$, then $z_{1} z_{2} \in I$. Hence $P_{1} P_{2} \subseteq I$.

Theorem 2.5. Let $I$ be a 2-absorbing ideal of $R$ such that $\operatorname{Rad}(I)=P$ is a prime ideal of $R$ and suppose that $I \neq P$. For each $x \in P \backslash I$ let $B_{x}=\{y \in R \mid y x \in I\}$. Then $B_{x}$ is a prime ideal of $R$ containing $P$. Furthermore, either $B_{y} \subseteq B_{x}$ or $B_{x} \subseteq B_{y}$ for every $x, y \in P \backslash I$.

Proof: Let $x \in P \backslash I$. Since $P^{2} \subseteq I$ (by Theorem 2.4), we conclude that $P \subseteq B_{x}$. Suppose that $P \neq B_{x}$ and $y z \in B_{x}$ for some $y, z \in R$. Since $P \subset B_{x}$, we may assume that $y \notin P$ and $z \notin P$, and thus $y z \notin I$. Since $y z \in B_{x}$, we have $y z x \in I$. Since $I$ is a 2-absorbing ideal of $R$ and $y z \notin I$, we conclude that either $y x \in I$ or $z x \in I$, and thus either $y \in B_{x}$ or $z \in B_{x}$. Hence $B_{x}$ is a prime ideal of $R$ containing $P$.

Let $x, y \in P \backslash I$ and suppose that $z \in B_{x} \backslash B_{y}$. Since $P \subseteq B_{y}, z \in B_{x} \backslash P$. We show that $B_{y} \subset B_{x}$. Let $w \in B_{y}$. Since $P \subseteq B_{x}$, we may assume that $w \in B_{y} \backslash P$. Since $z \notin P$ and $w \notin P$, we conclude that $z w \notin I$. Since $z(x+y) w \in I$ and $z w, z y \notin I$, we conclude that $(x+y) w \in I$. Hence $w x \in I$ since $(x+y) w \in I$ and $w y \in I$. Thus $w \in B_{y} \subseteq B_{x}$. $\square$

TheOREM 2.6. Let $I$ be a 2-absorbing ideal of $R$ such that $I \neq \operatorname{Rad}(I)=P_{1} \cap P_{2}$ where $P_{1}$ and $P_{2}$ are the only nonzero distinct prime ideals of $R$ that are minimal over I. Then for each $x \in \operatorname{Rad}(I) \backslash I, B_{x}=\{y \in R \mid x y \in I\}$ is a prime ideal of $R$ containing $P_{1}$ and $P_{2}$. Furthermore, either $B_{y} \subseteq B_{x}$ or $B_{x} \subseteq B_{y}$ for every $x, y \in \operatorname{Rad}(I) \backslash I$.

Proof: Let $x \in \operatorname{Rad}(I) \backslash I$. Since $P_{1} P_{2} \subseteq I$ by Theorem 2.4, we conclude that $x P_{1} \subseteq I$ and $x P_{2} \subseteq I$. Thus $P_{1} \subset B_{x}$ and $P_{2} \subset B_{x}$. Suppose $y z \in B_{x}$ for some $y, z \in R$. Since $P_{1} \subset B_{x}$ and $P_{2} \subset B_{x}$, we may assume that $y, z \notin P_{1}$ and $y, z \notin P_{2}$, and thus $y z \notin I$. Since $y z \in B_{x}$, we have $y z x \in I$. Since $I$ is a 2-absorbing ideal of $R$ and $y z \notin I$, we conclude that either $y x \in I$ or $z x \in I$, and thus either $y \in B_{x}$ or $z \in B_{x}$. Hence $B_{x}$ 
is a prime ideal of $R$. By using an argument similar to that in the proof of Theorem 2.5, one can easily complete the proof.

Recall that a nonzero proper ideal $I$ of a ring $R$ is said to be $Q$-primal if $Z(R / I)$ $=Q / I$ for some prime ideal $Q$ of $R$ containing $I$.

COROLlary 2.7. Suppose that $I$ is a 2-absorbing ideal of $R$ such that $I \neq$ $\operatorname{Rad}(I)$. Then $I$ is a $Q$-primal ideal of $R$ where $Q=\cup_{x \in \operatorname{Rad}(I) \backslash I} B_{x}$ (recall that $B_{x}=\{y \in$ $R \mid y x \in I\})$.

Proof: Let $a, b \in R \backslash I$ such that $a b \in I$. We show that $a, b \in B_{f}$ for some $f \in \operatorname{Rad}(I) \backslash I$. By Theorem 2.3, we conclude that either $\operatorname{Rad}(I)=P$ is a prime ideal of $R$ or $\operatorname{Rad}(I)=P_{1} \cap P_{2}$, where $P_{1}, P_{2}$ are the only distinct prime ideals of $R$ that are minimal over $I$. Suppose that $\operatorname{Rad}(I)=P$ is a prime ideal of $R$. Hence either $a \in P \backslash I$ or $b \in P \backslash I$, and thus either $a, b \in B_{a}$ or $a, b \in B_{b}$. Since $I \neq \operatorname{Rad}(I)$, $D=\left\{B_{x} \mid x \in \operatorname{Rad}(I) \backslash I\right\}$ is a set of linearly ordered (prime) ideals of $R$ by Theorem 2.5. Thus $Z(R / I)=\cup_{B_{x} \in D}\left(B_{x} / I\right)$ is an ideal of $R / I$.

Now suppose that $\operatorname{Rad}(I)=P_{1} \cap P_{2}$, where $P_{1}, P_{2}$ are the only distinct prime ideals of $R$ that are minimal over $I$. Since $a b \in \operatorname{Rad}(I)$, without loss of generality we may conclude that either $a \in \operatorname{Rad}(I) \backslash I$ or $a \in P_{1} \backslash P_{2}$ and $b \in P_{2} \backslash P_{1}$. If $a \in \operatorname{Rad}(I) \backslash I$, then $a, b \in B_{a}$. Suppose that $a \in P_{1} \backslash P_{2}$ and $b \in P_{2} \backslash P_{1}$. Since $I \neq \operatorname{Rad}(I)$, there is a $d \in \operatorname{Rad}(I) \backslash I$. Since $P_{1} \subset B_{d}$ and $P_{2} \subset B_{d}$ by Theorem 2.6, we have $a, b \in B_{d}$. Again, since $I \neq \operatorname{Rad}(I), D=\left\{B_{x} \mid x \in \operatorname{Rad}(I) \backslash I\right\}$ is a set of linearly ordered (prime) ideals of $R$ by Theorem 2.6. Thus $Z(R / I)=\cup_{B_{x} \in D}\left(B_{x} / I\right)$ is an ideal of $R / I$.

In Section 3, we give an example (see Example 3.12) of a $Q$-primal ideal $I$ of $R$ such that $\operatorname{Rad}(I)=P$ is a prime ideal of $R$ and $P^{2} \subset I$, but $I$ is not a 2-absorbing ideal of $R$.

THEOREM 2.8. Suppose that $I$ is an ideal of $R$ such that $I \neq \operatorname{Rad}(I)$ and $\operatorname{Rad}(I)$ is a prime ideal of $R$. Then the following statements are equivalent:

(1) $I$ is a 2-absorbing ideal of $R$;

(2) $B_{x}=\{y \in R \mid y x \in I\}$ is a prime ideal of $R$ for each $x \in \operatorname{Rad}(I) \backslash I$.

Proof: (1) $\Rightarrow(2)$. This is clear by Theorem 2.5.

$(2) \Rightarrow(1)$. Suppose that $x y z \in I$ for some $x, y, z \in R$. Since $\operatorname{Rad}(I)$ is a prime ideal of $R$, we may assume that $x \in \operatorname{Rad}(I)$. If $x \in I$, then $x y \in I$ and we are done. Hence assume that $x \in \operatorname{Rad}(I) \backslash I$. Thus $y z \in B_{x}$. Since $B_{x}$ is a prime ideal of $R$ by Theorem 2.5 , we conclude that either $y x \in I$ or $z x \in I$. Thus $I$ is a 2-absorbing ideal of $R$.

THEOREM 2.9. Let $I$ be an ideal of $R$ such that $I \neq \operatorname{Rad}(I)=P_{1} \cap P_{2}$ where $P_{1}$ and $P_{2}$ are nonzero distinct prime ideals of $R$ that are minimal over $I$. Then the following statement are equivalent:

(1) $I$ is a 2-absorbing ideal of $R$;

(2) $P_{1} P_{2} \subseteq I$ and $B_{x}=\{y \in R \mid y x \in I\}$ is a prime ideal of $R$ for each $x \in \operatorname{Rad}(I) \backslash I$. 
(3) $B_{x}=\{y \in R \mid y x \in I\}$ is a prime ideal of $R$ for each $x \in\left(P_{1} \cup P_{2}\right) \backslash I$.

Proof: $(1) \Rightarrow(2)$. This is clear by Theorems 2.4 and 2.6 .

$(2) \Rightarrow(3)$. Let $x \in P_{1} \backslash P_{2}$. It is clear that $y x \in I$ if and only if $y \in P_{2}$. Since $P_{1} P_{2} \subseteq I$, we conclude that $B_{x}=P_{2}$ is a prime ideal of $R$. Let $z \in P_{2} \backslash P_{1}$. By a similar argument as before we conclude that $B_{z}=P_{1}$ is a prime of $R$. Since $B_{d}$ is a prime ideal of $R$ for each $d \in \operatorname{Rad}(I) \backslash I$, we are done.

$(3) \Rightarrow(1)$. Let $x y z \in I$. We may assume that $x \in\left(P_{1} \cup P_{2}\right) \backslash I$. Thus $y z \in B_{x}$. Since $B_{x}$ is a prime ideal of $R$ by Theorem 2.6, we conclude that either $y x \in I$ or $z x \in I$, and hence $I$ is a 2-absorbing ideal of $R$.

THEOREM 2.10. Let $I$ be a 2 -absorbing ideal of a ring $R$ such that $I \neq \operatorname{Rad}(I)$. For each $x \in \operatorname{Rad}(I) \backslash I$, let $B_{x}=\{y \in R \mid y x \in I\}$. Then :

(1) If $x \in \operatorname{Rad}(I) \backslash I$ and $y \in R$ such that $y x \notin I$, then $B_{y x}=B_{x}$.

(2) If $x, y \in \operatorname{Rad}(I) \backslash I$ and $B_{x}$ is properly contained in $B_{y}$, then $B_{f x+d y}=B_{x}$ for every $f, d \in R$ such that $f d \notin B_{x}$. In particular, if $x, y \in \operatorname{Rad}(I) \backslash I$ and $B_{x}$ is properly contained in $B_{y}$, then $B_{x+y}=B_{x}$.

Proof: (1) Let $x, y \in \operatorname{Rad}(I) \backslash I$. Since $B_{x} \subset B_{y}$, it is clear that $B_{x} \subseteq B_{y x}$. Let $c \in B_{y x}$. Since $c y x \in I$, we conclude that $c y \in B_{x}$. Since $B_{x}$ is a prime ideal of $R$ by Theorems 2.5, 2.6 and $y \notin B_{x}$ because $y x \notin I$, we have $c x \in I$. Hence $c \in B_{x}$, and thus $B_{x}=B_{y x}$.

(2) Let $x, y \in \operatorname{Rad}(I) \backslash I$. Since $B_{x} \subset B_{y}$, it is clear that $B_{x} \subseteq B_{f x+d y}$. Suppose that $B_{x} \neq B_{f x+d y}$. Since $B_{x}, B_{f x+d y}, B_{y}$ are linearly ordered by Theorems $2.5,2.6$ and $B_{x}$ is properly contained in $B_{y}$, there is a $z \in B_{y} \cap B_{f x+d y}$ such that $z \notin B_{x}$. Since $z y \in I$ and $z(f x+d y) \in I$, we conclude that $z f x \in I$. Hence $z f \in B_{x}$, a contradiction since neither $z \in B_{x}$ nor $f \in B_{x}$. Thus $B_{x}=B_{f x+d y}$.

EXAMPLE 2.11. Suppose that $R=\mathcal{Z}[x, y]$ where $\mathcal{Z}$ is the ring of integers and $x, y$ are indeterminates, $P_{1}=(x, 2) R, P_{2}=(y, 2) R$ are prime ideals of $R$, and let $I=P_{1} P_{2}=$ $(4,2 x, 2 y, x y) R$. Then $\operatorname{Rad}(I)=P_{1} \cap P_{2}=(2, x y) R$. Since $B_{2}=\{z \in R \mid 2 z \in$ $I\}=(2, x, y) R$ is a (maximal) prime ideal of $R$, it is easy to see that $B_{d}=B_{2}$ for each $d \in \operatorname{Rad}(I) \backslash I$. Hence $I$ is a 2 -absorbing ideal of $R$ by Theorem 2.9 .

EXAMPLE 2.12. Suppose that $R=\mathcal{Z}[x, y, z]$ where $x, y, z$ are indeterminates, $P=(2, x) R$ is a prime ideal of $R$, and $I=\left(4,2 x, 2 y, x y, x z, x^{2}\right) R$. Then $P^{2} \subset I$ and $\operatorname{Rad}(I)=P$. Now $B_{2}=(2, x, y) R$ is a prime ideal of $R, B_{x}=(2, x, y, z) R$ is a (prime) maximal ideal of $R$, and $B_{2+x}=B_{2}$. It is easy to see that if $d \in P \backslash I$, then either $B_{d}=B_{2}$ or $B_{d}=B_{x}$. Thus $I$ is a 2-absorbing ideal of $R$ by Theorem 2.8. Observe that $I$ is not a primary ideal.

Part of this paper was presented at a commutative ring conference in Cortona, Italy (June, 2004). During the conference, Bruce Olberding asked the author the following 
question: Let $I$ be a 2-absorbing ideal of a ring $R$ and suppose that $I_{1} I_{2} I_{3} \subseteq I$ for some ideals $I_{1}, I_{2}, I_{3}$ of $R$, does it follow that $I_{1} I_{2} \subseteq I$ or $I_{2} I_{3} \subseteq I$ or $I_{1} I_{3} \subseteq I$ ? The answer to the question is yes as in the following result.

THEOREM 2.13. Suppose that $I$ is a nonzero proper ideal of a ring $R$. The following statements are equivalent:

(1) $I$ is a 2-absorbing ideal of $R$;

(2) If $I_{1} I_{2} I_{3} \subseteq I$ for some ideals $I_{1}, I_{2}, I_{3}$ of $R$, then $I_{1} I_{2} \subseteq I$ or $I_{2} I_{3} \subseteq I$ or $I_{1} I_{3} \subseteq I$.

Proof: Since (2) $\Rightarrow(1)$ is trivial, we only need to show that $(1) \Rightarrow(2)$. Suppose that $I_{1} I_{2} I_{3} \subseteq I$ for some ideals $I_{1}, I_{2}, I_{3}$ of $R$. By Theorem 2.4, we conclude that $\operatorname{Rad}(I)$ is a prime ideal of $R$ or $\operatorname{Rad}(I)=P_{1} \cap P_{2}$ where $P_{1}$ and $P_{2}$ are nonzero distinct prime ideals of $R$ that are minimal over $I$. If $I=\operatorname{Rad}(I)$, then it is easily proved that $I_{1} I_{2} \subseteq I$ or $I_{2} I_{3} \subseteq I$ or $I_{1} I_{3} \subseteq I$. Hence assume that $I \neq \operatorname{Rad}(I)$. We consider two cases.

Case I. Suppose that $\operatorname{Rad}(I)$ is a prime ideal of $R$. Then we may assume that $I_{1} \subseteq \operatorname{Rad}(I)$ and $I_{1} \notin I$. Let $x \in I_{1} \backslash I$. Since $x I_{2} I_{3} \subseteq I$, we conclude that $I_{2} I_{3} \subseteq B_{x}$. Since $B_{x}$ is a prime ideal of $R$ by Theorem 2.8, we conclude that either $I_{2} \subseteq B_{x}$ or $I_{3} \subseteq B_{x}$. If $I_{2} \subseteq B_{d}$ and $I_{3} \subseteq B_{d}$ for each $d \in I_{1} \backslash I$, then $I_{1} I_{2} \subseteq I$ (and $I_{1} I_{3} \subseteq I$ ) and we are done. Hence assume that that $I_{2} \subseteq B_{y}$ and $I_{3} \nsubseteq B_{y}$ for some $y \in I_{1} \backslash I$. Since $\left\{B_{w} \mid w \in I_{1} \backslash I\right\}$ is a set of prime ideals of $R$ that are linearly ordered by Theorem 2.5 and $I_{2} \subseteq B_{y}$ and $I_{3} \nsubseteq B_{y}$, we conclude that $I_{2} \subseteq B_{z}$ for each $z \in I_{1} \backslash I$, and thus $I_{1} I_{2} \subseteq I$.

Case II. Suppose that $\operatorname{Rad}(I)=P_{1} \cap P_{2}$ where $P_{1}$ and $P_{2}$ are nonzero distinct prime ideals of $R$ that are minimal over $I$. We may assume that $I_{1} \subseteq P_{1}$. If either $I_{2} \subseteq P_{2}$ or $I_{3} \subseteq P_{2}$, then either $I_{1} I_{2} \subseteq I$ or $I_{1} I_{3} \subseteq I$ because $P_{1} P_{2} \subseteq I$ by Theorem 2.4. Hence assume that $I_{1} \subseteq \operatorname{Rad}(I)$ and $I_{1} \nsubseteq I$. By an argument similar to that one given in case $I$ and Theorem 2.5, we are done.

\section{ON 2-ABSORBING IDEALS IN PARTICULAR CLASSES OF RINGS}

TheOREM 3.1. Suppose that $I$ is a $P$-primary ideal of a ring $R$. Then $I$ is a 2-absorbing ideal of $R$ if and only if $P^{2} \subseteq I$. In particular, $M^{2}$ is a 2-absorbing ideal of $R$ for each maximal ideal $M$ of $R$.

Proof: Suppose that $I$ is a 2-absorbing ideal of a ring $R$. Then $P^{2} \subseteq I$ by Theorem 2.4(1). Conversely, suppose that $P^{2} \subseteq I$ and $x y z \in I$. If either $x \in I$ or $y z \in I$, then there is nothing to prove. Hence assume that neither $x \in I$ nor $y z \in I$. Since $I$ is a $P$-primary ideal of $R$, we conclude that $x \in P$ and $y z \in P$. Thus $x, y \in P$ or $x, z \in P$. Since $P^{2} \subseteq I$, we conclude that $x y \in I$ or $x z \in I$.

Corollary 3.2. Suppose that $P$ is a nonzero prime ideal of $R$. Then $P^{(2)}=P^{2} R_{P} \cap R$ is a 2-absorbing ideal of $R$. 
Proof: It is well-known that $P^{(2)}$ is a $P$-Primary ideal of $R$. Since $P^{2} \subseteq P^{(2)}, P^{(2)}$ is a 2-absorbing ideal of $R$ by Theorem 3.1 .

The following lemma is useful in the proof of our next result.

LEMMA 3.3. Suppose that $R$ is a zero-dimensional ring with exactly two distinct maximal ideals such that $\operatorname{Nil}(R) \neq\{0\}, \operatorname{Nil}(R)^{2}=\{0\}$ and $\operatorname{Nil}(R)=w R$ for each nonzero $w \in \operatorname{Nil}(R)$. Then $R$ is ring-isomorphic to $R / M_{1}^{2} \oplus R / M_{2}$ where $M_{1}$ is a maximal ideal of $R$ such that $M_{1}^{2} \neq M_{1}$ and $M_{2}$ is a maximal of $R$ such that $M_{2}^{2}=M_{2}$. Furthermore, each nonzero proper ideal of $R$ is a 2-absorbing ideal of $R$.

PRoOF: Let $M_{1}, M_{2}$ be the two distinct maximal ideals of $R$. Since $\operatorname{Nil}(R)=M_{1} M_{2}$ and $\operatorname{Nil}(R)^{2}=\{0\}$, we conclude that $M_{1}^{2} M_{2}^{2}=\{0\}$. Since $M_{1}^{2}, M_{2}^{2}$ are co-maximal, $R$ is ring-isomorphic to $D=R / M_{1}^{2} \oplus R / M_{2}^{2}$. Since $\operatorname{Nil}(R) \neq\{0\}$, we conclude that at least one of the maximal ideals of $R$ is a non-idempotent ideal. Hence we may assume that $M_{1}^{2} \neq M_{1}$, and thus there is an element $m_{1} \in M_{1}$ such that $m_{1} \notin M_{1}^{2}$. Now suppose that $M_{2}^{2} \neq M_{2}$. Then there is an element $m_{2} \in M_{2}$ such that $m_{2} \notin M_{2}^{2}$. Since $\left(0, m_{2}\right.$ $\left.+M_{2}^{2}\right),\left(m_{1}+M_{1}^{2}, 0\right)$ are nonzero nilpotent elements of $D,\left(0, m_{2}+M_{2}^{2}\right) \in\left(m_{1}+M_{1}^{2}, 0\right) D$ by hypothesis, which is impossible. Thus $M_{2}^{2}=M_{2}$. Hence $\operatorname{Nil}(D)=\{0\} \oplus\left(M_{1} / M_{1}^{2}\right)$. Since $w D=\operatorname{Nil}(D)$ for each nonzero $w \in \operatorname{Nil}(D)$, we conclude that $\operatorname{Nil}(D)$ is the only proper non-maximal ideal of $D$. Thus every nonzero proper ideal of $D$ is a 2-absorbing ideal of $D$, and hence every nonzero proper ideal of $R$ is a 2-absorbing ideal of $R$.

Recall that an element $x \in R$ is said to be a $\pi$-regular element of $R$ if there is a positive integer $n$ and an element $y \in R$ such that $x^{2 n} y=x^{n}$. If every element of $R$ is a $\pi$-regular element, then $R$ is called a $\pi$-regular ring. It is well-known [4, Theorem 3.1] that a ring $R$ is a $\pi$-regular ring if and only if $R$ is a zero-dimensional ring.

THEOREM 3.4. Every nonzero proper ideal of a ring $R$ is a 2-absorbing ideal of $R$ if and only if $R$ is zero-dimensional (that is, $R$ is a $\pi$-regular ring) and one of the following statements hold:

(1) $R$ is quasi-local with maximal ideal $M=\operatorname{Nil}(R) \neq\{0\}$ such that $M^{2} \subseteq x R$ for each nonzero $x \in M$.

(2) $R$ has exactly two distinct maximal ideals such that either $R$ is ringisomorphic to $F_{1} \oplus F_{2}$ where $F_{1}$ and $F_{2}$ are fields or $\operatorname{Nil}(R)^{2}=\{0\}$ and $\operatorname{Nil}(R)=w R$ for each nonzero $w \in \operatorname{Nil}(R)$.

(3) $R$ is ring-isomorphic to $F_{1} \oplus F_{2} \oplus F_{3}$ where $F_{1}, F_{2}, F_{3}$ are fields.

Proof: Suppose that $R$ is quasi-local with maximal ideal $M=\operatorname{Nil}(R) \neq\{0\}$ such that $M^{2} \subseteq x R$ for each nonzero $x \in M$. Since every nonzero proper ideal $I$ of $R$ is an $M$-primary ideal of $R$ and $M^{2} \subseteq I$, we conclude that every nonzero proper ideal of $R$ is a 2-absorbing ideal of $R$ by Theorem 3.1. Suppose that $R$ is zero-dimensional and the second condition holds. If $\operatorname{Nil}(R)=\{0\}$, then it is easily proved that every nonzero proper ideal of $R$ is a 2-absorbing ideal of $R$. If $\operatorname{Nil}(R) \neq\{0\}$, then every nonzero proper 
ideal of $R$ is a 2-absorbing ideal of $R$ by Lemma 3.3. Suppose that $R$ is ring-isomorphic to $D=F_{1} \oplus F_{2} \oplus F_{3}$ where $F_{1}, F_{2}, F_{3}$ are fields. Since every nonzero proper ideal of $D$ is either a maximal ideal of $D$ or a product(intersection) of two distinct maximal ideals of $D$, we conclude that every nonzero proper ideal of $D$ is a 2-absorbing ideal of $D$, and hence every nonzero proper ideal of $R$ is a 2-absorbing ideal of $R$.

Conversely, suppose that every nonzero proper ideal of $R$ is a 2-absorbing ideal of $R$. We show that $R$ is a zero-dimensional ring. Let $w \in R$. If $w$ is a unit of $R$ or a nilpotent of $R$, then $w$ is a $\pi$-regular element of $R$. Hence assume that $w$ is a nonunit non-nilpotent element of $R$. Then $w^{4} R$ is a nonzero proper ideal of $R$, and hence it is a 2-absorbing ideal of $R$. Since $w^{4} \in w^{4} R$, we conclude that $w^{2} \in w^{4} R$, and thus $w$ is a $\pi$-regular element of $R$. Hence $R$ is a $\pi$-regular ring, and thus $R$ is a zero-dimensional ring.

Next we show that $R$ has at most three distinct maximal ideals. Suppose that $M_{1}, M_{2}, M_{3}$ are distinct maximal ideals of $R$. Then $I=M_{1} M_{2} M_{3}=M_{1} \cap M_{2} \cap M_{3}=\{0\}$, for if $I \neq\{0\}$, then $I=\operatorname{Rad}(I)$ is a 2-absorbing ideal of $R$ which is impossible by Theorem 2.4. Since $M_{1} M_{2} M_{3}=\{0\}, R$ has at most three distinct maximal ideals.

Now suppose that $R$ has exactly three distinct maximal ideal $M_{1}, M_{2}, M_{3}$. Since $M_{1} M_{2} M_{3}=\{0\}$, we conclude that $R$ is ring-isomorphic to $R / M_{1} \oplus R / M_{2} \oplus R / M_{3}$, and thus the third condition holds.

Suppose that $R$ has exactly two distinct maximal ideals $M_{1}, M_{2}$. If $\operatorname{Nil}(R)$ $=M_{1} M_{2}=\{0\}$, then $R$ is ring-isomorphic to $R / M_{1} \oplus R / M_{2}$. Hence assume that $\operatorname{Nil}(R)=M_{1} M_{2} \neq\{0\}$. Suppose that $\operatorname{Nil}(R)^{2} \neq\{0\}$. Then there are nonzero elements $w_{1}, w_{2} \in \operatorname{Nil}(R)$ such that $w_{1} w_{2} \neq 0$. Since $w_{1} w_{2} R$ is a 2-absorbing ideal of $R$, we conclude that $w_{1} \in M 1 M_{2}=\operatorname{Nil}(R) \subseteq w_{1} w_{2} R$ by Theorem 2.4. Hence $w_{1}=w_{1} w_{2} k$ for some nonzero $k \in R$, and thus $w_{1}\left(1-w_{2} k\right)=0$. Hence $w_{1}=0$ since $1-w_{2} k$ is a unit of $R$, a contradiction. Thus $\operatorname{Nil}(R)^{2}=\{0\}$. Suppose that $w$ is a nonzero nilpotent element of $R$. Since $w R$ is a 2-absorbing ideal of $R$, we conclude that $\operatorname{Nil}(R)=M_{1} M_{2} \subseteq w R$ by Theorem 2.4, and hence the second condition holds.

Finally suppose that $R$ is a quasi-local ring with maximal ideal $\operatorname{Nil}(R) \neq\{0\}$. Suppose that $w$ is a nonzero element of $\operatorname{Nil}(R)$. Since $w R$ is a 2-absorbing ideal of $R$, we conclude that $\operatorname{Nil}(R)^{2} \subseteq w R$ by Theorem 2.4. Thus the first condition holds.

EXAMPLE 3.5.

(a) Let $\mathcal{Z}$ be the ring of integers, $R=\mathcal{Z}_{8}$, and $D=\mathcal{Z}_{p^{2}} \oplus F$ where $p$ is a prime number of $\mathcal{Z}$ and $F$ is a field. Then every nonzero proper ideal of $R$ is a 2-absorbing ideal and every nonzero proper ideal of $D$ is a 2-absorbing ideal.

(b) Let $\mathcal{R}$ be the ring of all real numbers and $X, Y$ be indeterminates. Set $R=\mathcal{R}[[X, Y]] /\left(X Y, X^{2}-Y^{2}, X^{3}, Y^{3}\right)$. Then every nonzero proper ideal of $R$ is a 2-absorbing ideal. 
Recall that a prime ideal of $R$ is called a divided prime if $P \subset(x)$ for every $x \in R \backslash P$.

THEOREM 3.6. Suppose that $P$ is a nonzero divided prime ideal of $R$ and $I$ is an ideal of $R$ such that $\operatorname{Rad}(I)=P$. Then the following statements are equivalent:

(1) I is a 2-absorbing ideal of $R$;

(2) $I$ is a $P$-primary ideal of $R$ such that $P^{2} \subseteq I$.

Proof: $(1) \Rightarrow(2)$. Suppose that $I$ is a 2-absorbing ideal of $R$. Since $\operatorname{Rad}(I)=P$ is a nonzero prime ideal of $R, P^{2} \subseteq I$ by Theorem 2.4(1). Now let $x y \in I$ for some $x, y \in R$ and suppose that $y \notin P$. Since $x \in P$ and $P$ is a divided ideal of $R$, we conclude that $x=y k$ for some $k \in R$. Hence $x y=y^{2} k \in I$. Since $y^{2} \notin I$ and $I$ is a 2-absorbing ideal of $R$, we conclude that $y k=x \in I$. Thus $I$ is a $P$-primary ideal of $R$.

(2) $\Rightarrow(1)$. This is clear by Theorem 3.1 .

THEOREM 3.7. Suppose that $\operatorname{Nil}(R)$ and $P$ are divided prime ideals of a ring $R$ such that $P \neq \operatorname{Nil}(R)$. Then $P^{2}$ is a 2-absorbing ideal of $R$.

Proof: First we observe that $\operatorname{Nil}(R) \subset P^{2}$ since $P \neq \operatorname{Nil}(R)$ and $\operatorname{Nil}(R)$ is divided. By Theorem 3.6 it suffices to show that $P^{2}$ is a $P$-primary ideal of $R$. Suppose that $x y=p_{1} q_{1}+\cdots+p_{n} q_{n} \in P^{2}$ where the $p_{i}$ 's and the $q_{i}$ 's are in $P$, and suppose that $y \notin P$. Since $P$ is a divided ideal of $R$, we conclude that $x y=y c_{1} q_{1}+\cdots+y c_{n} q_{n} \in P^{2}$ where the $c_{i}$ 's are in $P$. Hence $y\left(x-c_{1} q_{1}-\cdots-c_{n} q_{n}\right)=0 \in \operatorname{Nil}(R)$. Since $y \notin \operatorname{Nil}(R)$ (because $y \notin P$ ) and $\operatorname{Nil}(R)$ is a prime ideal of $R$, we conclude that $x-c_{1} q_{1}-\cdots-c_{n} q_{n}=w \in \operatorname{Nil}(R)$. Since $\operatorname{Nil}(R) \subset P^{2}$, we conclude that $x=c_{1} q_{1}+\cdots c_{n} q_{n}+w \in P^{2}$, and thus $P^{2}$ is a $P$-primary ideal of $R$.

If $R$ is an integral domain, then $\operatorname{Nil}(R)=\{0\}$ is a divided prime ideal of $R$. Hence we have the following corollary.

COROLlaRY 3.8. Suppose that $P$ is a a nonzero divided prime ideal of an integral domain $R$. Then $P^{2}$ is a 2-absorbing ideal of $R$.

The following is an example of a prime ideal $P$ of an integral domain $R$ such that $P^{2}$ is not a 2-absorbing ideal of $R$.

EXAmple 3.9. Suppose that $R=\mathcal{Z}+6 x \mathcal{Z}[x]$ and $P=6 x \mathcal{Z}[x]$ (where $\mathcal{Z}$ is the ring of integers and $x$ is an indeterminate). Then $P$ is a prime ideal of $R$. Since $6 x^{2} \in P \backslash P^{2}$ and $B_{6 x^{2}}=\left\{y \in R \mid 6 x^{2} y \in P^{2}\right\}=6 \mathcal{Z}+6 x \mathcal{Z}[x]$ is not a prime ideal of $R, P^{2}$ is not a 2-absorbing ideal of $R$ by Theorem 2.8 .

Proposition 3.10. Suppose that $R$ is a valuation domain and $I$ is a nonzero proper ideal of $R$. Then the following statements are equivalent:

(1) $I$ is a 2-absorbing ideal of $R$;

(2) $I$ is a a $P$-primary ideal of $R$ such that $P^{2} \subseteq I$;

(3) $I=P$ or $I=P^{2}$ where $P=\operatorname{Rad}(I)$ is a prime ideal of $R$. 
Proof: $(1) \Rightarrow(2)$. Suppose that $I$ is a 2-absorbing ideal of $R$. Then $\operatorname{Rad}(I)=P$ is a prime ideal of $R$. Since $R$ is a divided domain, $I$ is a $P$-primary ideal of $R$ such that $P^{2} \subseteq I$ by Theorem 3.6.

(2) $\Rightarrow(3)$. Suppose that $I$ is a $P$-primary ideal of $R$ such that $P^{2} \subseteq I$. Since $R$ is a valuation domain, we conclude that either $I=P$ or $I=P^{2}$ by [5, Theorem 5.11, p. 106].

(3) $\Rightarrow(1)$. Suppose that either $I=P$ or $I=P^{2}$ where $P=\operatorname{Rad}(I)$ is a prime ideal of $R$. If $I=P$, then $I$ is a 2-absorbing ideal of $R$. If $I=P^{2}$, then $I$ is a 2-absorbing ideal of $R$ by Corollary 3.8 .

The following is an example of a prime ideal $P$ of an integral domain $R$ such that $P^{2}$ is a 2-absorbing ideal of $R$, but $P^{2}$ is not a $P$-primary ideal of $R$.

EXAMPLE 3.11. Suppose that $R=\mathcal{Z}+3 x \mathcal{Z}[x]$ (where $\mathcal{Z}$ is the ring of integers and $x$ is an indeterminate) and let $P=3 x \mathcal{Z}[x]$ be a prime ideal of $R$. Since $3\left(3 x^{2}\right) \in P^{2}$, we conclude that $P^{2}$ is not a $P$-primary ideal of $R$. It is easy to verify that if $d \in P \backslash P^{2}$, then either $B_{d}=\{y \in R \mid y d \in I\}=P$ or $B_{d}=3 \mathcal{Z}+3 x \mathcal{Z}[x]$ is a prime ideal of $R$. Hence $P^{2}$ is a 2 -absorbing ideal by Theorem 2.8 .

Next we show that for each $n \geqslant 2$, there is a valuation domain $R$ with maximal ideal $M$ and Krull dimension $n$ that admits an $M$-primal ideal $I$ such that $\operatorname{Rad}(I)=P$ is a prime ideal of $R, P^{2} \subset I$, and the Krull dimension of $R / I$ is $n-1$, but $I$ is not a 2-absorbing ideal of $R$.

EXAMPLE 3.12. Suppose that $n \geqslant 2$ and $D$ be a valuation domain with quotient field $K$ and Krull dimension $n-1$. Let $X$ be an indeterminate and set $R=D+X K[[X]]$. Then $R$ is a valuation domain with Krull dimension $n$. Let $P=X K[[X]]$ be a prime ideal of $R$ and let $Q$ be a nonzero prime ideal of $R$ such that $Q \neq P$. Then it is clear that $P \subset Q$. Set $I=X R_{Q}$. Then $I$ is an ideal of $R$ such that $\operatorname{Rad}(I)=P$ and $Z(R / I)=Q / I$ by [1, Proposition 2.1]. Hence $I$ is not a primary ideal of $R$. Since $R$ is a valuation domain and $X \in P \backslash P^{2}$, we have $P^{2} \subset I$ and $I$ is not a 2-absorbing ideal of $R$ by Proposition 3.10. By construction it is clear that the Krull dimension of $R / I$ is $n-1$.

Before we state our next theorem, the following lemma is needed.

LEMMA 3.13. Suppose that $I$ is a 2-absorbing ideal of a ring $R$ and let $S$ be a multiplicatively closed subset of $R$. If $I R_{S} \neq\{0\}$, then $I R_{S}$ is a 2-absorbing ideal of $R_{S}$.

Proof: Suppose that $x y z \in I R_{S}$ for some $x, y, z \in R_{S}$. Then there are elements $s \in S$, and $x_{1}, x_{2}, x_{3} \in R$ such that $x y z=\left(x_{1} / s\right)\left(x_{2} / s\right)\left(x_{3} / s\right)=x_{1} x_{2} x_{3} / s^{3} \in I R_{S}$. Thus, $x_{1} x_{2} x_{3} \in I$. Since $I$ is a 2-absorbing ideal of $R$, we have $x_{1} x_{2} \in I$ or $x_{1} x_{3} \in I$ or $x_{2} x_{3} \in I$, and thus $x y \in I R_{S}$ or $x z \in I R_{S}$ or $y z \in I R_{S}$.

Theorem 3.14. Suppose that $R$ is a Prüfer domain and $I$ is a nonzero ideal of $R$. Then the following statements are equivalent:

(1) $I$ is a 2-absorbing ideal of $R$; 
(2) $I$ is a prime ideal of $R$ or $I=P^{2}$ is a $P$-primary ideal of $R$ or $I=P_{1} \cap P_{2}$ where $P_{1}$ and $P_{2}$ are nonzero prime ideals of $R$.

Proof: Suppose that $I$ is a nonzero 2-absorbing ideal of $R$. Then either $\operatorname{Rad}(I)=P$ is a prime ideal of $R$ or $\operatorname{Rad}(I)=P_{1} \cap P_{2}$ where $P_{1}, P_{2}$ are the only minimal prime ideals of $R$ over $I$ by Theorem 2.4. Suppose that $\operatorname{Rad}(I)=P$ is a prime ideal of $R$ and $I \neq P$. Then $I$ is a $Q$-primal ideal of $R$ by Corollary 2.7 , and $P \subseteq Q$ because $P^{2} \subseteq I$ by Theorem 2.4. Since $I R_{Q}$ is a 2-absorbing ideal of $R_{Q}$ by Lemma 3.13 and $R_{Q}$ is a valuation domain, we conclude that $I R_{Q}$ is a $P R_{Q}$-primary ideal of $R_{Q}$ by Proposition 3.10. Hence $I R_{Q} \cap R$ is a $P$-primary ideal of $R$ by $[5$, Corollary 3.10, p. 68]. It is easy to verify that $I R_{Q} \cap R=I$ (for a proof see [2, Lemma 1.3]). Hence $I=I R_{Q} \cap R$ is a $P$-primary ideal of $R$. Since $P^{2} \subseteq I$ by Theorem 2.4 and $I \neq P$, we conclude that $I=P^{2}$ by [5, Proposition $6.9(4)$, p. 132].

Next suppose that $\operatorname{Rad}(I)=P_{1} \cap P_{2}$ where $P_{1}, P_{2}$ are the only minimal prime ideals of $R$ over $I$. Assume that $I \neq \operatorname{Rad}(I)$. Then $I$ is a $Q$-primal ideal of $R$ by Corollary 2.7. Since $P_{1} \subset Q$ and $P_{2} \subset Q$ and $R_{Q}$ is a valuation domain, either $P_{1} R_{Q} \subset P_{2} R_{Q}$ or $P_{2} R_{Q} \subset P_{1} R_{Q}$, which is impossible. Thus $I=\operatorname{Rad}(I)=P_{1} \cap P_{2}$.

For the converse, just observe that if $I=P^{2}$ is a $P$-primary ideal of $R$, then $I$ is a 2-absorbing ideal of $R$ by Theorem 3.1.

Recall that an integral domain $R$ is said to be a Dedekind domain if every nonzero ideal of $R$ is invertible.

THEOREM 3.15. Let $R$ be a Noetherian domain that is not a field. The following statements are equivalent:

(1) $R$ is a Dedekind domain;

(2) If $I$ is a 2-absorbing ideal of $R$, then $I$ is a maximal ideal of $R$ or $I=M^{2}$ for some maximal ideal $M$ of $R$ or $I=M_{1} M_{2}$ where $M_{1}, M_{2}$ are some maximal ideals of $R$;

(3) If $I$ is a 2-absorbing ideal of $R$, then $I$ is a prime ideal of $R$ or $I=P^{2}$ for some prime ideal $P$ of $R$ or $I=P_{1} \cap P_{2}$ where $P_{1}, P_{2}$ are some prime ideals of $R$.

Proof: $(1) \Rightarrow(2)$. Since $R$ is a one-dimensional ring, every nonzero prime ideal of $R$ is maximal. Suppose that $I$ is a 2-absorbing ideal of $R$. Then either $\operatorname{Rad}(I)=M$ is a a maximal ideal of $R$ or $\operatorname{Rad}(I)=M_{1} \cap M_{2}=M_{1} M_{2}$ for some distinct maximal ideals $M_{1}, M_{2}$ of $R$ by Theorem 2.4 .

$(2) \Rightarrow(3)$. This is obvious.

$(3) \Rightarrow(1)$. Let $M$ be a maximal ideal of $R$. Since every ideal between $M^{2}$ and $M$ is an $M$-Primary ideal and hence a 2-absorbing ideal of $R$ by Theorem 3.1, the hypothesis in (3) implies that there are no ideals properly between $M^{2}$ and $M$. Hence $R$ is a Dedekind domain by $[3$, Theorem 39.2 , p. 470$]$. 
Recall that an integral domain $R$ is said to be an almost Dedekind domain if $R_{M}$ is a Dedekind domain for each maximal ideal $M$ of $R$ (that is, $R_{M}$ is a Noetherian valuation domain for each maximal ideal $M$ of $R$ and hence $R$ is a one-dimensional ring.) The following result is a characterisation of an almost Dedekind domain in terms of 2absorbing ideals. The proof of the following result is similar to the proof of Theorem 3.15 , and hence it is left to the reader.

Proposition 3.16. Let $R$ be an integral domain that is not a field and suppose that $R_{M}$ is Noetherian for each maximal ideal $M$ of $R$. The following statements are equivalent:

(1) $R$ is an almost Dedekind domain;

(2) If $I$ is a 2-absorbing ideal of $R$, then $I$ is a maximal ideal of $R$ or $I=M^{2}$ for some maximal ideal $M$ of $R$ or $I=M_{1} M_{2}$ where $M_{1}, M_{2}$ are some maximal ideals of $R$;

(3) If $I$ is a 2-absorbing ideal of $R$, then $I$ is a prime ideal of $R$ or $I=P^{2}$ for some prime ideal $P$ of $R$ or $I=P_{1} \cap P_{2}$ where $P_{1}, P_{2}$ are some prime ideals of $R$.

\section{REFERENCES}

[1] A. Badawi, 'On $\phi$-pseudo-valuation rings, II', Houston J. Math. 26 (2000)), 473-480.

[2] L. Fuchs, W. Heinzer, and B. Olberding, 'Commutative ideal theory without finiteness conditions: primal ideals', Tran. Amer. Math. Soc. (to appear).

[3] R. Gilmer, Multiplicative ideal theory, Queen's Papers Pure Appl. Math. 90 (Queen's University, Kingston, 1992).

[4] J. Huckaba, Commutative rings with zero divisors (Marcel Dekker, New York, Basel, 1988).

[5] M.D. Larson and P.J. McCarthy, Multiplicative theory of ideals (Academic Press, New York, London, 1971).

Department of Mathematics and Statistics

American University Of Sharjah

P.O. Box 26666

Sharjah

United Arab Emirates

e-mail: abadawi@aus.edu 